International Journal of Biomedicine | June 2021 - Volume 11, Issue Suppl_1: Abstracts from the Third Russian International Conference "Cryo-electron microscopy 2021: achievements and prospects"

POSTER ABSTRACT PRESENTATIONS

SESSION TITLE: EM RESEARCH RELATED TO MEDICINE

DOI: 10.21103/IJBM.11.Suppl_1.P40

\title{
Abstract P-40: The Shape and Size of the Recombinant Virus-like Particles Were Checked by Means of Electron Microscope
}

\author{
$\underline{\text { Ivan Okhrimenko }}^{1}$, Yulia Zagryadskaya $^{1}$, Alexey Tsarenko ${ }^{1}$, \\ Valentin Borshchevskiy ${ }^{1}$, Andrey Rogachev ${ }^{1}$, Marina Shevchenko ${ }^{2}$ \\ ${ }^{1}$ Moscow Institute of Physics and Technology (MIPT), Dolgoprudny, Russia \\ ${ }^{2}$ Shemyakin and Ovchinnikov Institute of Bioorganic Chemistry, Moscow, Russia
}

Background: Nucleocapsid protein of hepatitis-B virus ( $\mathrm{HBcAg}$ ) recombinantly synthesized in prokaryotic and eukaryotic cells is known to be capable to selfassemble into highly immunogenic stable viral-like particles (VLP) of icosahedral shape with a characteristic size of $32 \mathrm{~nm}$ (Schödel et al., 1994; Murray and Shiau, 1999). The VLP formation is tolerant to the insertion of some artificial epitopes to $\mathrm{N}$ - and $\mathrm{C}$-termini of $\mathrm{HBcAg}$ monomer and also into major insertion region (MIR), forming a spike on the surface of VLP (Tordjeman et al., 1993, Peyret et al., 2015).

Methods: We have investigated the possibility of heterologous expression of $d e$ novo designed gene coding the first 148 amino acid residues of $\mathrm{HBcAg}$ (Pumpens and Grens, 1999). The gene was specially designed to be suitable for the insertions of genes coding fluorescent proteins, which are desired for the studies of VLP distribution in tissues by confocal microscopy. Gene was optimized for overexpression in E. coli producer strains and special attention was taken to obtain a simple purification scheme, which reliably reduces the amount of pyrogens in purified VLP.

The MIPT scientific platform of electron microscopy equipped with the transmission electron microscope Tecnai Polara G2 (Thermo Scientific (FEI)) was used. Carbon-coated (Lacey Carbon and $10 \mathrm{~nm}$ thin carbon) copper 200 mesh grids were treated with glow-discharge and coated with VLP suspension in deionized water. The samples were stained with uranyl acetate solution, airdried, and inspected at the accelerating voltage of $300 \mathrm{kV}$.

Results: The $32 \mathrm{~nm}$ size of heterologously synthesized VLP was successfully proved, and spherical shape was seen using negative contrasting.

Key Words: hepatitis-B virus $・$ electron microscopy $・$ VLP 
This work was supported by the Russian Foundation for Basic Research (Grant No. 20-04-60311)

*Corresponding author: Ivan Okhrimenko.E-mail: ivan.okhrimenko@phystech.edu

International Journal of Biomedicine. 2021;11 Suppl 1: S29-30.

doi: 10.21103/IJBM.11.Suppl_1.P40

(C)2021 International Medical Research and Development Corporation 